\title{
Pupillographic Sleepiness Test and Polysomnography in Nondemented Patients with Ischemic White Matter Lesions
}

\author{
Ralf Landwehr' ${ }^{1}$ and Robert Liszka ${ }^{2}$ \\ ${ }^{1}$ Neurologische Klinik, Pfalzklinikum für Psychiatrie und Neurologie, Teaching Hospital, Medical Department, \\ University of Mainz, 76889 Klingenmünster, Germany \\ ${ }^{2}$ Klinik für Neurologie, Westpfalz-Klinikum, Teaching Hospital, Medical Departments, \\ Universities of Mainz and Heidelberg/Mannheim, 67655 Kaiserslautern, Germany
}

Correspondence should be addressed to Ralf Landwehr; ralf_landwehr@web.de

Received 18 August 2015; Revised 9 November 2015; Accepted 11 November 2015

Academic Editor: Stephen D. Ginsberg

Copyright (C) 2015 R. Landwehr and R. Liszka. This is an open access article distributed under the Creative Commons Attribution License, which permits unrestricted use, distribution, and reproduction in any medium, provided the original work is properly cited.

\begin{abstract}
Background. Patients with ischemic white matter lesions (WML) frequently report nonrestorative sleep or daytime sleepiness. However, subjective estimations of sleep and sleepiness can differ considerably from objective measures. The pupillographic sleepiness test (PST) could identify patients with sleep disorders requiring polysomnography (PSG) and further treatment. Methods. We performed a PST and a PSG of 35 nondemented patients with WML, who reported nonrestorative sleep or daytime sleepiness, and assessed the diagnostic value of the pupillary unrest index (PUI). Sleep parameters were compared to normative data. Results. The mean PUI of WML patients was normal $(5.89 \mathrm{~mm} / \mathrm{min})$ and comparable to PUIs of patients with other neurological disorders. All 9 of the 35 WML patients (25.7\%) who had a PUI above normal also had clinically relevant sleep disorders (5: sleep apnea, 7: periodic leg movements, and 4: insomnia). Six patients with a normal PUI had mild to moderate primary insomnia. Conclusion. PST and PSG parameters were physiological in most patients with WML. Age-related changes and affective and neuropsychological disorders might account for their sleep-related complaints. An elevated PUI in patients with WML seems to indicate comorbid sleep disorders that require further diagnostic evaluation and treatment (sleep apnea, insomnia with periodic leg movements, but not primary insomnia).
\end{abstract}

\section{Introduction}

White matter lesions (WML) are a common finding in cerebral MRI scans of elderly persons [1, 2]. However, their clinical and pathophysiological significance remains a matter of debate. The majority of WML are due to microvascular ischemic lesions caused mainly by arterial hypertension and other common vascular risk factors like diabetes mellitus, hypercholesterolemia, and smoking. Although elderly persons with WML can appear clinically asymptomatic, neuropsychological testing can reveal impairments of processing speed, immediate and delayed memory, and executive function [1]. The presentation of clinical symptoms is mostly subacute resulting from the combination of nonocclusive small vessel disease and lacunes [3], with a chronic course marked by fluctuations and slowly progressive worsening [4].
Typical symptoms are loss of frontal executive functions, reduced information processing speed, gait disturbances, depressive mood, motivation and initiative loss, and urinary incontinence. Without sufficient treatment, the disease might ultimately develop to vascular dementia.

Up to date, data about objective sleep parameters in patients with ischemic WML are scarce. In general, cognitive impairment and sleep disorders tend to aggravate each other mutually [5-7]. A recently published study [8] described several differences of polysomnographic parameters in patients with vascular cognitive impairment but without dementia, patients with simple stroke, and controls with similar comorbidities but without stroke: compared with controls, both patient groups had reduced total sleep time (TST), slow wave sleep (SWS), REM sleep, and sleep efficiency, a longer sleep onset latency, and an increased arousal index. 
In addition, various sleep disturbances and increased daytime sleepiness in patients with WML have been described and were further assessed using questionnaires [9]. Other studies have reported similar symptoms and disorders after both cortical and subcortical ischemic stroke $[8,10,11]$. Sleeprelated breathing disorders can be a consequence of stroke and represent an independent risk factor for cerebrovascular diseases [12,13]. Stroke-related sleep disturbances are very common during the acute phase and persist in $25 \%$ of the patients [12]. Frequent complaints about sleep-wakedisorders with nonrestorative sleep and daytime sleepiness as core symptoms suggest comparable problems in patients with ischemic WML. However, the subjective estimation and objective measures of sleepiness can differ considerably, especially in subjects with sleep disorders [14]. Moreover, the neuropsychological impairments of patients with WML could reduce the ability to assess sleep-related symptoms correctly, as was shown for patients with mild cognitive impairment [15]. Affective disorders might also mimic sleeprelated symptoms. Lastly, the quality and recreational effects of sleep tend to decrease with age $[16,17]$. Polysomnography (PSG) is the gold standard to identify sleep disorders that require treatment. On the other hand, PSG is not always available, time- and personnel-consuming, and expensive. Alternative objective screening measures like the pupillographic sleepiness test (PST) are therefore required to distinguish pathological sleepiness from age-related, neuropsychological, and affective impairments.

The pupillographic sleepiness test is an efficient and easily applicable method to assess the level of the central nervous activation. It is based on the autonomous regulation of the pupillary diameter in the absence of light, which dilates with sympathetic and constricts with parasympathetic innervation. At a physiological arousal level, the central sympathicotonus is high and inhibits the parasympathetic nucleus Edinger-Westphal of the N. oculomotorius. Thus, the diameter of the pupil remains nearly constant for a long time, with only minor changes around $1 \mathrm{~Hz}$. In a state of sleepiness, however, fluctuations of the pupillary diameter increase over time, due to a decreasing effect of the sympathicus on the pupil directly and on the nucleus Edinger-Westphal $[18,19]$. Fluctuations especially in the frequency range below $0.8 \mathrm{~Hz}$ correlate with the variations of the central nervous activation, so these were termed "fatigue waves."

The objective of the present study was the identification and quantification of sleep disorders in patients with WML without dementia, based on complaints of nonrestorative sleep or daytime sleepiness. In order to assess the diagnostic value of the PST in these patients, we used the PST as a screening procedure and evaluated the result by a one-night PSG. Considering the physiological principle of the PST, we expected to identify patients with sleep disorders causing increased daytime sleepiness, like sleep apnea, periodic limb movements in sleep, and potentially secondary (symptomatic) insomnia. Since PST measures in primary insomnia are usually within the normal range [20], a discrimination of patients with primary insomnia and patients with secondary insomnia (which is more prevalent in elderly persons) might be possible.

\section{Materials and Methods}

2.1. Participants. Our study was based on a retrospective analysis of clinical and PSG data of 35 patients with WML who suffered from nonrestorative sleep (32 patients) or daytime sleepiness ( 9 patients); 6 patients reported both of these symptoms. The mean age of the patients was $72.6 \pm 4.30$ years (64.1-87.4); 19 were male and 16 female. 34 patients (97\%) had cardiovascular risk factors: 31 : arterial hypertension (89\%), 25: hypercholesterolemia with LDL >130 mg/dL (71\%), 9: diabetes mellitus (26\%), 4: active smoking (11\%), and 16: a history of smoking (47\%). WML were diagnosed by cerebral MRI scans on a single $1.5 \mathrm{~T}$ scanner (Magnetom, Siemens Medical Solutions, Erlangen, Germany) with the following sequences: axial T1-weighted, T2-weighted, FLAIR, and DWI, coronal T1-weighted, and sagittal T2-weighted (axial T2*-weighted sequences were additionally performed when hemorrhagic lesions needed to be excluded). We estimated the amount of WML with the rating scale of Manolio et al. [21]. This scale is widely accepted, and its correlation with other visual rating scales is high [22]. Cognitive deficits were evaluated by a CERAD (27 patients) or MMST (8 patients). Cutoff values were 85 (CERAD) and 28 (MMST) for mild cognitive impairment and 77 (CERAD) and 25 (MMST) for dementia [23]. Only patients with normal neuropsychological test results or mild cognitive impairment (28 and 7 patients, resp.) were included in the study. Sleep disorders were diagnosed according to clinical criteria from a detailed anamnesis supplemented by sleep questionnaires (Pittsburgh Sleep Quality Index "PSQI," Epworth Sleepiness Scale "ESS"), a pupillographic sleepiness test, and one night of polysomnography. The cutoff values were $>5$ for the PSQI and $>9$ for the ESS; these scores were applied to complement the clinical criteria based on the medical history and not for diagnosis. Exclusion criteria were previous treatment for the respective sleep disorders, the intake of medications or substances with central nervous effects, concomitant or former neurological and psychiatric disorders (especially a history of stroke or the detection of territorial infractions in the MRI), and abnormal findings in the waking EEG (21 leads). All participants had given informed consent for their EEG data to be used for further evaluation. The local ethics committee had approved this procedure.

2.2. Polysomnography. Participants underwent one-night inlab videopolysomnographic sleep recordings (Schwarzer Brainlab 3.30-0.00, Belgium) including the following leads: EEG (F3-A2, F4-A1, C3-A2, C4-A1, O1-A2, and O2-A1), according to the international 10-20-system, Ag-AgClelectrodes (TC $0.3 \mathrm{~s}, \mathrm{HF} 70 \mathrm{~Hz}$, and sampling rate $250 \mathrm{~Hz}$ ), electrooculogram, electromyography of submental muscles, left and right tibial muscles, oral and nasal airflow measured by thermistor, thoracic and abdominal excursions by plethysmography, snoring by microphone, blood oxygen saturation by finger oximetry, and ECG by a D2 lead and body position. The recordings were stored on hard disc. Visual sleep scoring [24] by two sleep specialists was based on $30 \mathrm{~s}$ epochs of the sleep EEG, EOG, and EMG. Since there was no control group, the results of the PSG were compared to epidemiologic 
TABLE 1: Sleep parameters of WML patients with normal PUI $\left(P_{\text {nor }}, n=26\right)$ and elevated PUI $\left(P_{\text {elev }}, n=9\right)$.

\begin{tabular}{|c|c|c|c|c|c|}
\hline \multirow[b]{2}{*}{ Sleep parameter } & \multirow{2}{*}{$\begin{array}{c}P_{\text {nor }}: \text { normal PUI } \\
(4.32 \mathrm{~mm} / \mathrm{min}), n=26\end{array}$} & \multicolumn{4}{|c|}{$P_{\text {elev }}:$ elevated PUI $(10.76 \mathrm{~mm} / \mathrm{min}), n=9$} \\
\hline & & $\begin{array}{c}\text { Sleep apnea } \\
(13.08 \mathrm{~mm} / \mathrm{min}), n=5\end{array}$ & $\begin{array}{l}\text { Significance of } \\
\text { differences to } P_{\text {nor }}\end{array}$ & $\begin{array}{c}\text { Insomnia } \\
(7.86 \mathrm{~mm} / \mathrm{min}), n=4\end{array}$ & $\begin{array}{l}\text { Significance of } \\
\text { differences to } P_{\text {nor }}\end{array}$ \\
\hline Total sleep time & $6.3 \pm 1.1 \mathrm{~h}$ & $6.6 \pm 1.6 \mathrm{~h}$ & n.s. & $5.5 \pm 1.3 \mathrm{~h}$ & n.s. \\
\hline Sleep onset latency & $16.5 \pm 15.8 \mathrm{~min}$ & $11.3 \pm 13.7 \mathrm{~min}$ & n.s. & $27.4 \pm 24.0 \mathrm{~min}$ & 0.05 \\
\hline REM latency & $98.4 \pm 40.6 \mathrm{~min}$ & $146.2 \pm 55.2 \mathrm{~min}$ & 0.05 & $129.8 \pm 51.7 \mathrm{~min}$ & 0.05 \\
\hline WASO & $6.8 \pm 2.9 \%$ & $12.7 \pm 8.7 \%$ & 0.05 & $16.9 \pm 15.9 \%$ & 0.05 \\
\hline N1 sleep & $7.1 \pm 4.5 \%$ & $18.9 \pm 11.5 \%$ & 0.05 & $16.3 \pm 12.4 \%$ & 0.05 \\
\hline N2 sleep & $59.6 \pm 9.1 \%$ & $51.5 \pm 14.0 \%$ & n.s. & $58.7 \pm 17.0 \%$ & n.s. \\
\hline Slow wave sleep & $7.4 \pm 7.7 \%$ & $2.1 \pm 1.8 \%$ & 0.05 & $0.0 \pm 0.0 \%$ & 0.01 \\
\hline REM sleep & $19.2 \pm 8.4 \%$ & $14.8 \pm 6.4 \%$ & n.s. & $8.2 \pm 12.2 \%$ & 0.05 \\
\hline AHI & $3.0 \pm 2.3 / \mathrm{h}$ & $33.6 \pm 15.2 / \mathrm{h}$ & 0.001 & $6.1 \pm 3.9 / \mathrm{h}$ & n.s. \\
\hline PMLS-Index & $6.2 \pm 5.0 / \mathrm{h}$ & $17.7 \pm 12.6 / \mathrm{h}$ & 0.01 & $27.8 \pm 9.8 / \mathrm{h}$ & 0.05 \\
\hline
\end{tabular}

The $P_{\text {elev }}$ group was separated by the apnea-hypopnea-index (AHI) into an apnea-subgroup and an insomnia-subgroup. WASO = wake after sleep onset, AHI = apnea-hypopnea-index, and PMLS = periodic leg movements in sleep.

data from the literature $[6,17,25]$. An apnea-hypopneaindex $>5.0 / \mathrm{h}$ and a PLMS-index $>5.0 / \mathrm{h}$ were considered as increased, with an AHI $>10 / \mathrm{h}$ as definitely pathological [24].

2.3. Pupillographic Sleepiness Test. We used a standardized pupillographic measurement protocol with the PST xsII (AMTech $\mathrm{GmbH}$, Dossenheim, Germany): the pupillary diameter was registered for 11 minutes in the sitting subject with an infrared camera under quiet conditions and light shielding, between 8:00 and 11:00 a.m. on the day before the PSG. The pupillary unrest index (PUI) is based on the sum of the absolute changes of the pupil diameter (in $\mathrm{mm} / \mathrm{min}$ ) and relates to cumulative changes of the pupil diameter over the measurement period. It was automatically calculated in $40 \mathrm{~ms}$ bin quantities by the PST device's standard software. Normative data of the PUI exist for male and female adults at different ages $[18,26]$ : a physiological PUI is $<6.6 \mathrm{~mm} / \mathrm{min}$ (the mean PUI of the normative study $+1 \mathrm{SD}$ ), a borderline PUI between 6.6 and $9.8 \mathrm{~mm} / \mathrm{min}$ (mean PUI $\geq+1$ SD to $<$ $+2 \mathrm{SD}$ ), and a pathological PUI $\geq 9.8 \mathrm{~mm} / \mathrm{min}$ (mean PUI + $\geq 2 \mathrm{SD}$ ). In our study, all PUIs $\geq 6.6 \mathrm{~mm} / \mathrm{min}$ are designated as "elevated," since, in this range, further diagnostics are required. The PUI was also compared to patients with other neurological disorders from our data bank [27].

2.4. Statistics. All statistical analyses were performed by a PC-based statistical program (MacAnova 5.05, release 3, University of Minnesota, 2006). When a normal distribution of variables seemed probable, this was confirmed by the Kolmogorov-Smirnov-test. Differences between normally distributed variables were assessed using the $t$-test. In not normally distributed parameters, the significance of differences was assessed using the Wilcoxon-Mann-Whitneytest. Correlations were calculated according to the Pearson's correlation coefficient for normally distributed variables. Significance was assumed for $P \leq 0.05$.

\section{Results}

The mean value for the Manolio global rating of all 35 patients was $3.6 \pm 2.3$ (median 3 ), ranging from 0 to 8 ; based on this rating, most patients had mild or moderate WML. The mean PUI $=5.89 \pm 3.59 \mathrm{~mm} / \mathrm{min}$ of WML patients was physiological. 26 patients had a normal PUI (mean $4.32 \pm$ $1.07 \mathrm{~mm} / \mathrm{min}$, range $2.52-6.22 \mathrm{~mm} / \mathrm{min}$ ); 13 of these patients were male and 13 female. Nine patients ( 6 male, 3 female) had an elevated PUI $=10.76 \pm 4.06 \mathrm{~mm} / \mathrm{min}$. The range of individual PUI values was large from 6.75 to $18.12 \mathrm{~mm} / \mathrm{min}$; only 5 patients ( 4 male, 1 female) had a definitely pathological PUI $\geq 9.80 \mathrm{~mm} / \mathrm{min}$.

The mean ESS score of all patients was $6.4 \pm 3.9$ (range 317 ), and the mean PSQI score was $10.1 \pm 4.0$ (range 5-20); the patients with a normal PUI had an ESS score of $5.9 \pm 4.3$ and a PSQI score of $9.8 \pm 3.7$, and the patients with an elevated PUI had an ESS score of 7.0 \pm 6.1 and a PSQI score of $10.5 \pm 4.4$. None of these differences reached significance.

We analyzed the PSG results of the 26 patients with a normal PUI $\left(P_{\text {norm }}\right)$ and the 9 patients with an elevated PUI $\left(P_{\text {elev }}\right)$ separately (Table 1$) . P_{\text {norm }}$ results regarding latencies, global sleep parameters, and the distribution of sleep stages were comparable to the sleep heart health study data [17]. The mean AH- and PLMS-indices of $P_{\text {norm }}$ were also normal. We found gender differences only for sleep onset latencies (men $21.3 \mathrm{~min}$, women $11.7 \mathrm{~min}, P<0.05$ ) and REM latencies (men $117.8 \mathrm{~min}$, women $79.0 \mathrm{~min}, P<0.05$ ) but not for the other PSG parameters and the PUI. Only $6 P_{\text {norm }}$ patients were clinically diagnosed with mild to moderate primary insomnia; all these patients had corresponding alterations of PSG parameters: prolonged sleep onset and REM latencies, increased proportions of waking after sleep onset, and light sleep (N1, N2), reduced to absent slow wave sleep (N3), and more arousal than normal sleepers. This patient group had a mean PUI of $3.49 \pm 0.93$. No $P_{\text {norm }}$ patient had sleep apnea or PLMS. 
The $P_{\text {elev }}$ group was not homogenous in regard to sleep disorders: sleep apnea was diagnosed in $5 P_{\text {elev }}$ patients with a mean $\mathrm{AHI}=33.6 / \mathrm{h}: 4$ had obstructive and 1 mixed sleep apnea ( 3 of these patients also had PLMS). Four $P_{\text {elev }}$ patients had insomnia, as diagnosed by clinical and PSG criteria. Based on these diagnoses, we divided the $P_{\text {elev }}$ group into two subgroups with sleep apnea and insomnia, respectively. The PUI of the sleep apnea-subgroup $(13.08 \mathrm{~mm} / \mathrm{min})$ was higher than the PUI of the insomnia-subgroup $(7.86 \mathrm{~mm} / \mathrm{min}, P<$ 0.05). PSG data of both subgroups differed from the $P_{\text {norm }}$ group by an increased amount of wake after sleep onset and stage N1, reduced slow wave sleep, a prolonged REM sleep latency, and an elevated AHI and PLMS-index (Table 1). All 4 of the insomnia patients and 3 of the patients with obstructive sleep apnea had PLMS. The insomnia patients with an elevated PUI had also reduced REM sleep. Neither REM behavior disorders nor other parasomnias were diagnosed in the $P_{\text {norm }}$ and $P_{\text {elev }}$ groups. $P_{\text {elev }}$ patients had significantly more vascular risk factors than $P_{\text {norm }}$ (3.1 versus 1.7, $P<0.05$ ). The PSG parameters for insomnia patients from the $P_{\text {norm }}$ and the $P_{\text {elev }}$ groups differed only for the PLMS-index $(5.1 / \mathrm{h}$ versus $27.8 / \mathrm{h}$ ), but not for sleep latencies and stages.

In summary, all patients with an elevated PUI had clinically relevant sleep disorders, while only 6 patients of $26(23 \%)$ with a normal PUI had mild to moderate primary insomnia, but no other sleep disorders. Therefore, the PUI separated normal sleepers from patients with sleep apnea and patients with insomnia plus PLMS, but not from patients with primary insomnia without PLMS. This can also be expressed by the predictive values for the PUI with a cutoff value of $6.60 \mathrm{~mm} / \mathrm{min}$ : for the $P_{\text {elev }}$ group (sleep apnea or insomnia plus PLMS), the positive predictive value (PPV) and the negative predictive values (NPV) were 1.0, respectively; when considering all sleep disorders including primary insomnia, the PPV was also 1.0 and the NPV 0.77. A cutoff value of $9.80 \mathrm{~mm} / \mathrm{min}$ reduced the PPV to 0.56 and the NPV to 0.87 (including primary insomnia NPV 0.67). Only 3 of 5 sleep apnea patients and 2 of 4 insomnia plus PLMS patients had an ESS score $>9$, while 6 patients with physiological PSG and PST measures also had an ESS score $>9$. All 9 patients in the $P_{\text {elev }}$ group and 24 of 26 patients in the $P_{\text {norm }}$ group had a PSQI score $>5$.

The PSG parameters and the PUI did not differ between patients with normal cognitive function and patients with mild cognitive impairment. The percentage of SWS and REM sleep correlated negatively with the value of the Manolio scale, so a larger amount of WML implied a reduced percentage of SWS and REM sleep: Pearson's correlation coefficient was $r=-0.43(P<0.05)$ for SWS and $r=-0.37(P<0.05)$ for REM sleep and the Manolio score, respectively.

\section{Discussion}

Our study had two main objectives: Firstly, we examined patients with WML complaining of nonrestorative sleep or daytime sleepiness using the PST and PSG, in order to complement the scarce data about objective sleep parameters of patients with WML in the literature. Secondly, we assessed the sensitivity of the PST regarding the identification of WML patients who suffer from clinically relevant sleep disorders, based on the polysomnographic sleep parameters. Most of our patients with ischemic WML, in spite of their complaints of nonrestorative sleep or daytime sleepiness, had a normal PUI and normal objective sleep parameters in PSG, comparable to patients of the sleep heart health study [17] and normal sleepers of the same age $[6,25]$. However, all patients with an elevated PUI had clinically relevant sleep disorders; the PUI distinguished healthy sleepers from patients with sleep apnea and from insomnia patients with PLMS, but not from insomnia patients without PLMS. This result is in accordance with previously published data, documenting that patients with primary insomnia tend to have normal PST values [28]. Since the patients with an elevated PUI had relevant sleep disorders as well as significantly more vascular risk factors, the PST might be an important screening technique to identify a subgroup of patients with WML at high risk of vascular events. This result is crucial especially for patients with sleep apnea, since sleep-related breathing disorders themselves increase the risk of cardiovascular and cerebrovascular pathology [12, 13]. Furthermore, the PST distinguished insomnia patients with PLMS from insomnia patients without PLMS, so the PLMS might further reduce sleep quality in insomnia patients [29]. Interestingly, a cutoff value of $6.60 \mathrm{~mm} / \mathrm{min}$ for the PUI had a better predictive value for relevant sleep disorders than the definitely pathological cutoff value of $9.80 \mathrm{~mm} / \mathrm{min}$. Whether this result correlates to the smaller pupil diameter in the examined age group or other age-related changes can not be distinguished by our data. PLMS are a common finding in patients with sleep apnea and tend decrease with successful treatment of the apneas [30], although treatment might also unmask PLMS. Since treatment effects of sleep apnea were not registered in our study, we did not make a distinction between sleep apnea patients with and without PLMS.

Normal aging seems to involve qualitative and quantitative changes in sleep parameters, though with a high degree of individuality in the sleep/wake patterns [31]: epidemiologic studies have consistently described a reduction of TST and sleep efficiency, a minor decrease of REM sleep, and in men a substantial reduction of SWS $[6,17,25]$. The PSG sleep parameters in our study confirmed these results for the examined age group (64-87 years). When discussing sleep parameters of geriatric populations, the influence of comorbidities on sleep has to be taken into account, healthy persons without any diagnosis being rare in this age group. Since our patients had mostly typical cardiovascular risk factors, they could represent a realistic sample of geriatric patients, despite the selection bias caused by WML and subjective sleep disturbances. Alterations of sleep parameters in patients with vascular cognitive impairment [8] and stroke $[8,10,11]$ are qualitatively similar but considerably more pronounced. The negative correlation of the quantity of WML (scored by the Manolio scale) with the percentage of SWS and REM sleep is in good accordance with the sleep changes Jiang et al. [8] described for patients with vascular cognitive impairment; $46 \%(26 / 56)$ of these also had diffuse white matter hyperintensities.

The mean PUI of our 35 patients was comparable to patients with other neurological disorders who had 
a PUI $=6.22 \pm 3.17 \mathrm{~mm} / \mathrm{min}$, range $2.52-18.12 \mathrm{~mm} / \mathrm{min}$ [27] If only the 26 patients with a normal PUI are considered, their mean PUI of $4.32 \mathrm{~mm} / \mathrm{min}$ was only slightly higher than the mean PUI $(3.98 \mathrm{~mm} / \mathrm{min})$ of a group of healthy sleepers aged $60-79 y$ in the normative study of Eggert et al. [18]. Since the 6 of our patients with a normal PUI and primary insomnia had a mean PUI of $3.49 \mathrm{~mm} / \mathrm{min}$, they cannot account for this difference. However, compared to the younger groups (20-60 y) in the studies of Wilhelm et al. [26] and Eggert et al. [18] with a mean PUI of $4.50 \mathrm{~mm} / \mathrm{min}$ and $4.58 \mathrm{~mm} / \mathrm{min}$, respectively, our 26 patients had a lower PUI. Thus, the age effect on the PUI described by Eggert et al. is also confirmed by our data, even if less distinct.

Sleep disturbances seem to be less prominent in patients with WML compared to patients with MCI, probably due to the disruption of multiple intracortical networks by $\mathrm{A} \beta$ aggregation in the latter group [15]. In contrast, the ability to assess the sleep duration and restorative effects correctly seems to be similarly impaired in both patient groups, since the majority of our patients complained of nonrestorative sleep and daytime sleepiness, in spite of having age-appropriate PST and PSG parameters.

Limitations of our study were as follows: We had no control group, so we compared our PSG results to three large epidemiologic studies $[6,17,25]$ and our PST results to normative data [26] that provide data for the examined age group. Further methodical limitations are the retrospective study design, a selection bias due to the selective examination of inpatients without dementia, and the 1st-night effect of PSG, since only 1 night of PSG was available. Next, most of our patients had mild to moderate WML, so no conclusions concerning patients with severe WML were possible. Lastly, WML are a common finding in cerebral MRI scans of elderly persons. The clinical significance of WML, however, remains a matter of debate, with contradictory literature on the topic, due to several reasons [2]. (1) WML might be an incidental finding, especially due to the increasing sensitivity of MRI devices. (2) Several terms are used in the literature with inexact definitions and mutual discrimination (referred to as cerebral microangiopathy, encephalomalacia, leukoaraiosis, M. Binswanger, subcortical arteriosclerotic encephalopathy, and vascular dementia). (3) Different etiologies may contribute to WML, although most cases seem to be caused by cerebral small vessel ischemic disease. (4) The correlation of WML and clinical symptoms is not always definite. Therefore, our collective of WML patients might be heterogeneous, although the majority had multiple vascular risk factors as the most probable cause of WML.

Why had most examined patients age-appropriate PSG and PST parameters in contrast to their subjective impairments of sleep and vigilance? As subjective and objective measures of sleep quality and sleepiness can differ considerably [18], confounding factors concerning the self-assessment of sleep quality and daytime sleepiness need to be taken into account: apathy, abulia, and depression are common in patients with cerebrovascular disorders [31] and may present with overlapping or coexisting clinical symptoms [32]. Especially in patients with major depressive disorder, high incidences of hyperintense lesions in periventricular and deep white matter as well as subcortical and brain stem areas have been described [33]. Nondemented patients with WML also tend to have reduced global cognitive functioning and more specific neuropsychological deficits of processing speed, memory, and executive function [1]. One may assume that all these impairments might affect the self-assessment of sleep quality and daytime sleepiness. In addition, the deterioration of sleep quality and quantity with age itself, combined with a poor sleep perception [8], might cause an overestimation of sleep problems and thus the subjective experience of nonrestorative sleep. This hypothesis is supported by the low predictive values of the ESS and PSQI in our study, since not all patients with sleep apnea and daytime sleepiness had an elevated ESS, and a relevant number of patients with physiological PSG and PST parameters had increased ESS and PSQI scores. Lastly, the distinction of fatigue and sleepiness is difficult for many patients suffering from insomnia; the sympathicotonus is frequently elevated in these patients, so PUI values are often conspicuously low [20]. The PST results of the 6 insomnia patients in our study are in accordance with these findings.

In conclusion, PST and PSG parameters were physiological in most patients with WML despite their complaints of nonrestorative sleep or daytime sleepiness. Primary insomnia, age-related changes, and affective and neuropsychological disorders might therefore contribute to these subjective impairments. However, an elevated PUI seems to be a strong indicator of comorbid sleep disorders causing increased daytime sleepiness (sleep apnea, insomnia plus periodic leg movements) and possibly also of patients at higher risk of vascular events. The PST is an efficient and easily applicable method to assess daytime sleepiness in WML patients and could thus facilitate a preselection of those patients that require a PSG and potentially a medical treatment of their sleep disorders and associated vascular risk factors. However, the PST is not suited to identification of subjects with insomnia (without PLMS) who might also need a PSG.

\section{Conflict of Interests}

The authors declare that there is no conflict of interests regarding the publication of this paper.

\section{References}

[1] F. M. Gunning-Dixon and N. Raz, "The cognitive correlates of white matter abnormalities in normal aging: a quantitative review," Neuropsychology, vol. 14, no. 2, pp. 224-232, 2000.

[2] D. J. Moser, J. E. Kanz, and K. D. Garrett, "White matter hyperintensities and cognition," in Vascular Dementia: Cerebrovascular Mechanics and Clinical Management, R. H. Paul, R. Cohen, B. R. Ott, and S. Salloway, Eds., pp. 223-229, Humana Press, Totowa, NJ, USA, 2005.

[3] G. C. Román, T. Erkinjuntti, A. Wallin, L. Pantoni, and H. C. Chui, "Subcortical ischaemic vascular dementia," The Lancet Neurology, vol. 1, no. 7, pp. 426-436, 2002.

[4] G. C. Román, "Clinical forms of vascular dementia," in Vascular Dementia: Cerebrovascular Mechanisms and Clinical 
Management, R. H. Paul, R. Cohen, B. R. Ott, and S. Salloway, Eds., pp. 7-21, Humana Press, Totowa, NJ, USA, 2005.

[5] G. D’Onofrio, D. Sancarlo, F. Panza et al., "Neuropsychiatric symptoms and functional status in alzheimer's disease and vascular dementia patients," Current Alzheimer Research, vol. 9, no. 6, pp. 759-771, 2012.

[6] T. E. Gildner, M. A. Liebert, P. Kowal, S. Chatterji, and J. J. Snodgrass, "Associations between sleep duration, sleep quality, and cognitive test performance among older adults from six middle income countries: results from the study on global ageing and adult health (SAGE)," Journal of Clinical Sleep Medicine, vol. 10, no. 6, pp. 613-621, 2014.

[7] K. Meguro, M. Ueda, I. Kobayashi et al., "Sleep disturbance in elderly patients with cognitive impairment, decreased daily activity and periventricular white matter lesions," Sleep, vol. 18, no. 2, pp. 109-114, 1995.

[8] B. Jiang, C. Ding, G. Yao et al., "Polysomnographic abnormalities in patients with vascular cognitive impairment-nodementia," Sleep Medicine, vol. 14, no. 11, pp. 1071-1075, 2013.

[9] C.-Y. Cheng, C.-F. Tsai, S.-J. Wang, C.-Y. Hsu, and J.-L. Fuh, "Sleep disturbance correlates with white matter hyperintensity in patients with subcortical ischemic vascular dementia," Journal of Geriatric Psychiatry and Neurology, vol. 26, no. 3, pp. 158164, 2013.

[10] M. M. Siccoli, N. Rölli-Baumeler, P. Achermann, and C. L. Bassetti, "Correlation between sleep and cognitive functions after hemispheric ischaemic stroke," European Journal of Neurology, vol. 15, no. 6, pp. 565-572, 2008.

[11] A. Terzoudi, T. Vorvolakos, I. Heliopoulos, M. Livaditis, K. Vadikolias, and H. Piperidou, "Sleep architecture in stroke and relation to outcome," European Neurology, vol. 61, no. 1, pp. 1622, 2009.

[12] M. Manconi, I. Zavalko, C. Cereda et al., "Longitudinal polysomnographic assessment from acute to subacute phase in infratentorial versus supratentorial stroke," Cerebrovascular Diseases, vol. 37, no. 2, pp. 85-93, 2014.

[13] M. C. Valenza, A. Baranchuk, G. Valenza-Demet, T. MuñozCasaubon, J. A. Martin-Navajas, and J. Healey, "Prevalence of risk factors for atrial fibrillation and stroke among 1210 patients with sleep disordered breathing," International Journal of Cardiology, vol. 174, no. 1, pp. 73-76, 2014.

[14] M. T. Bianchi, W. Wang, and E. B. Klerman, "Sleep misperception in healthy adults: implications for insomnia diagnosis," Journal of Clinical Sleep Medicine, vol. 8, no. 5, pp. 547-554, 2012.

[15] E. Hita-Yañez, M. Atienza, and J. L. Cantero, "Polysomnographic and subjective sleep markers of mild cognitive impairment," Sleep, vol. 36, no. 9, pp. 1327-1334, 2013.

[16] J. R. D. Espiritu, "Aging related sleep changes," Clinics in Geriatric Medicine, vol. 24, no. 1, pp. 1-14, 2008.

[17] M. L. Unruh, S. Redline, M.-W. An et al., "Subjective and objective sleep quality and aging in the sleep heart health study," Journal of the American Geriatrics Society, vol. 56, no. 7, pp. 12181227, 2008.

[18] T. Eggert, C. Sauter, R. Popp, J. Zeitlhofer, and H. Danker-Hopfe, "The pupillographic sleepiness test in adults: effect of age, gender, and time of day on pupillometric variables," American Journal of Human Biology, vol. 24, no. 6, pp. 820-828, 2012.

[19] E. R. Samuels and E. Szabadi, "Functional neuroanatomy of the noradrenergic locus coeruleus: its roles in the regulation of arousal and autonomic function part II: physiological and pharmacological manipulations and pathological alterations of locus coeruleus activity in humans," Current Neuropharmacology, vol. 6, no. 3, pp. 254-285, 2008.

[20] K. L. Lichstein, N. M. Wilson, S. L. Noe, R. N. Aguillard, and S. N. Bellur, "Daytime sleepiness in insomnia: behavioral, biological and subjective indices," Sleep, vol. 17, no. 8, pp. 693702, 1994.

[21] T. A. Manolio, R. A. Kronmal, G. L. Burke et al., "Magnetic resonance abnormalities and cardiovascular disease in older adults: The Cardiovascular Health study," Stroke, vol. 25, no. 2, pp. 318-327, 1994.

[22] L. Pantoni, M. Simoni, G. Pracucci, R. Schmidt, F. Barkhof, and D. Inzitari, "Visual rating scales for age-related white matter changes (leukoaraiosis): can the heterogeneity be reduced?" Stroke, vol. 33, no. 12, pp. 2827-2833, 2002.

[23] M. J. Chandler, L. H. Lacritz, L. S. Hynan et al., "A total score for the CERAD neuropsychological battery," Neurology, vol. 65, no. 1, pp. 102-106, 2005.

[24] M. H. Silber, S. Ancoli-Israel, M. H. Bonnet et al., "The visual scoring of sleep in adults," Journal of Clinical Sleep Medicine, vol. 3, no. 2, pp. 121-131, 2007.

[25] S. Redline, H. L. Kirchner, S. F. Quan, D. J. Gottlieb, V. Kapur, and A. Newman, "The effects of age, sex, ethnicity, and sleepdisordered breathing on sleep architecture," Archives of Internal Medicine, vol. 164, no. 4, pp. 406-418, 2004.

[26] B. Wilhelm, A. Körner, K. Heldmaier, K. Moll, H. Wilhelm, and H. Lüdtke, "Normwerte des pupillographischen Schläfrigkeitstests für Frauen und Männer zwischen 20 und 60 Jahren," Somnologie, vol. 5, no. 3, pp. 115-120, 2001.

[27] R. Landwehr, R. Woessner, M. Stoll, B. Endres, and J. Treib, "The pupillographic sleepiness test in patients with subcortical arteriosclerotic encephalopathy," Cerebrovascular Diseases, vol. 29, supplement 2, p. 292, 2010.

[28] D. M. Claman, S. K. Ewing, S. Redline, S. Ancoli-Israel, J. A. Cauley, and K. L. Stone, "Study of Osteoporotic Fractures Research Group. Periodic leg movements are associated with reduced sleep quality in older men: the MrOS Sleep Study," Journal of Clinical Sleep Medicine, vol. 15, no. 9, pp. 1109-1117, 2013.

[29] D. L. Bliwise, J. S. Carroll, and W. C. Dement, "Predictors of observed sleep/wakefulness in residents in long-term care," Journals of Gerontology, vol. 45, no. 4, pp. M126-M130, 1990.

[30] S. Aritake-Okada, K. Namba, N. Hidano et al., "Change in frequency of periodic limb movements during sleep with usage of continuous positive airway pressure in obstructive sleep apnea syndrome," Journal of the Neurological Sciences, vol. 317, no. 1-2, pp. 13-16, 2012.

[31] M. E. Mahler and J. L. Cummings, "Behavioral neurology of multi-infarct dementia," Alzheimer Disease and Associated Disorders, vol. 5, no. 2, pp. 122-130, 1991.

[32] A. Finset and S. Andersson, "Coping strategies in patients with acquired brain injury: relationships between coping, apathy, depression and lesion location," Brain Injury, vol. 14, no. 10, pp. 887-905, 2000.

[33] H. A. Sackeim, "Brain structure and function in late-life depression," in Advances in Brain Imaging, J. M. Morihisa, Ed., pp. 83-122, American Psychiatric Publishing, Washington, DC, USA, 2001. 


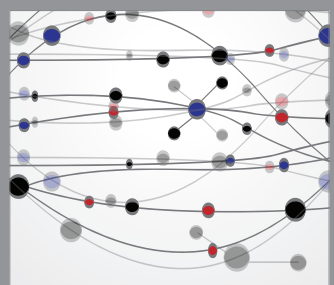

The Scientific World Journal
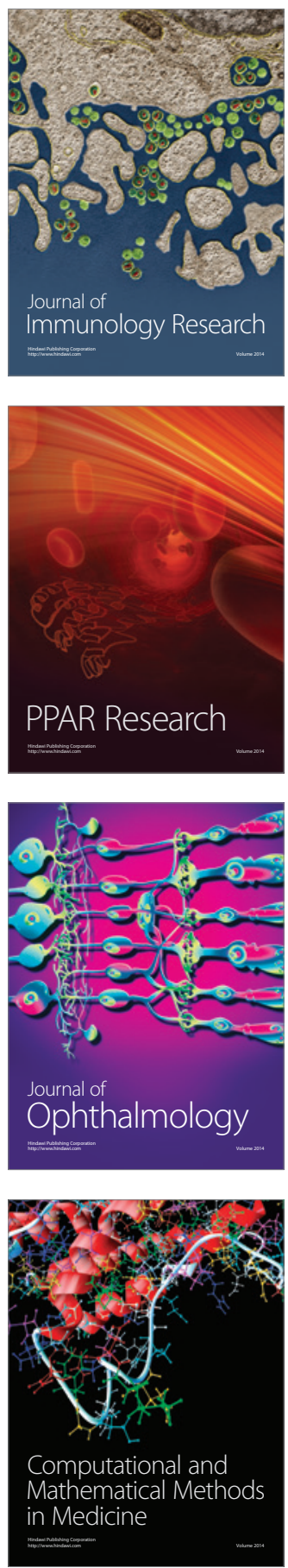

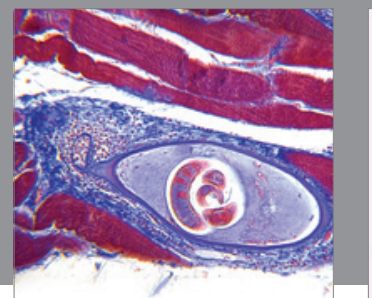

Gastroenterology

Research and Practice
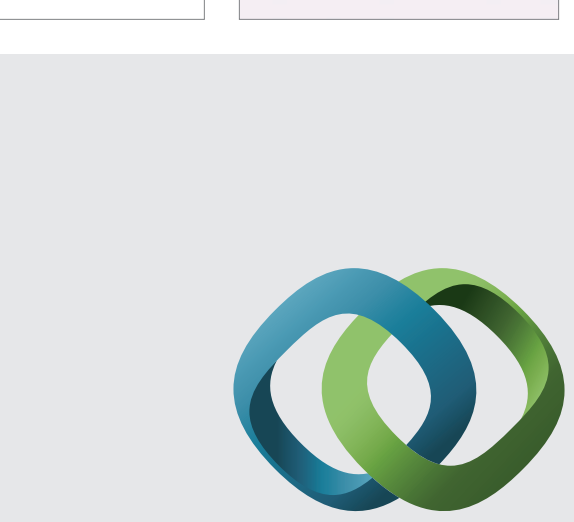

\section{Hindawi}

Submit your manuscripts at

http://www.hindawi.com
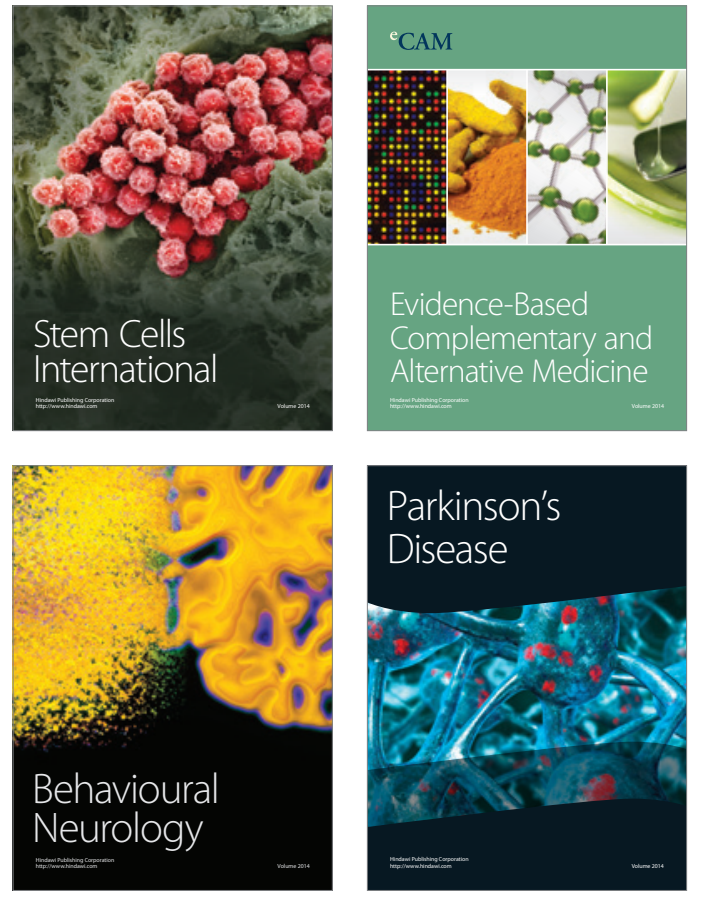
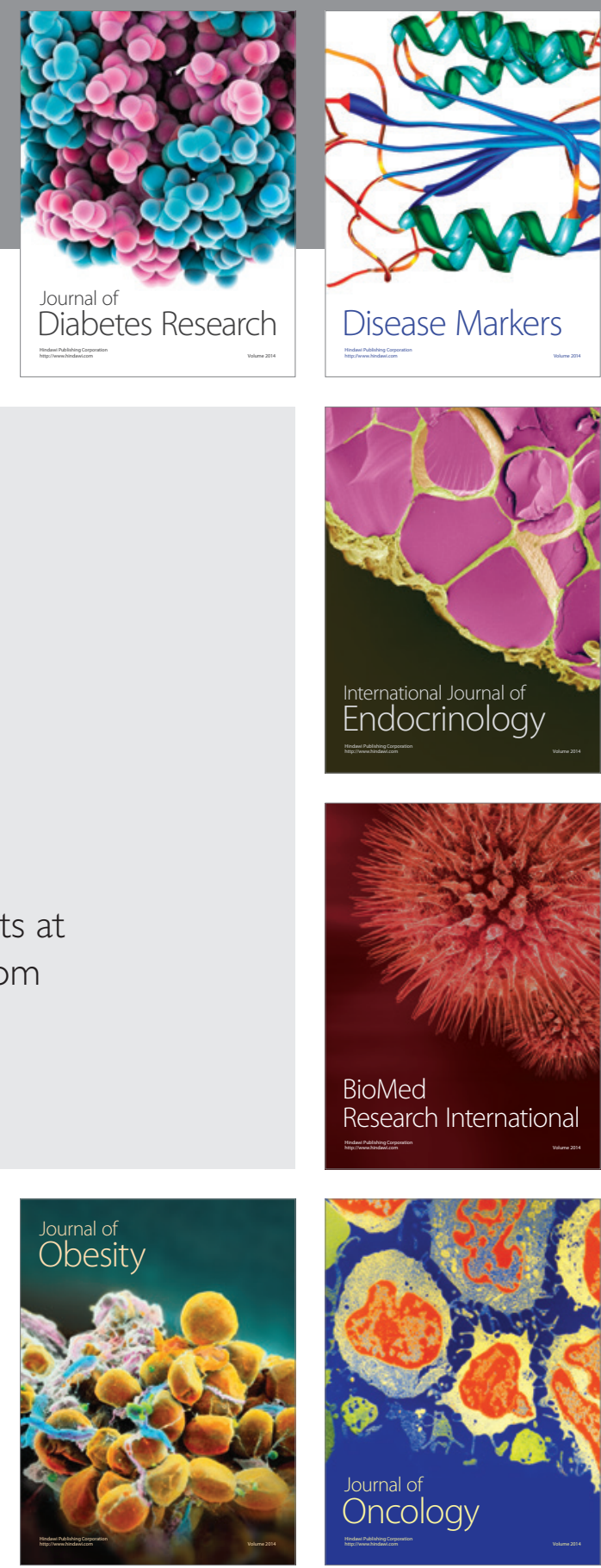

Disease Markers
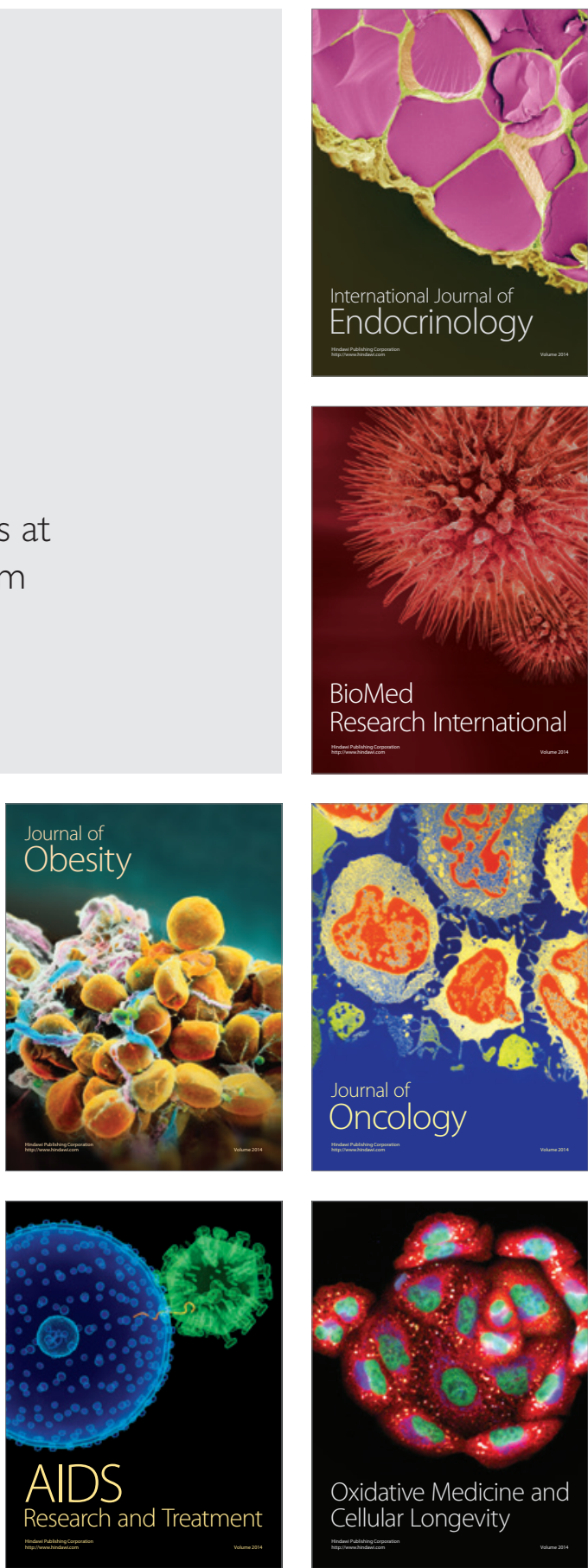